\title{
Astaxanthin blocks preeclampsia progression by suppressing oxidative stress and inflammation
}

\author{
RONG-RONG XUAN ${ }^{1}$, TING-TING NIU ${ }^{2}$ and HAI-MIN CHEN ${ }^{2}$ \\ ${ }^{1}$ Department of Gynecology and Obstetrics, The Affiliated Hospital of Medical College of Ningbo University, \\ Ningbo, Zhejiang 315020; ${ }^{2}$ School of Marine Science, Ningbo University, Ningbo, Zhejiang 315211, P.R. China
}

Received August 5, 2015; Accepted July 15, 2016

DOI: $10.3892 / \mathrm{mmr} .2016 .5569$

\begin{abstract}
To investigate the antioxidative effect of astaxanthin on $\mathrm{N} \omega$-nitro-L-arginine methyl ester (L-NAME)-induced preeclamptic rats. Cell survival, the level of reactive oxygen species (ROS) and the changes in mitochondrial membrane potential (MMP) were examined in astaxanthin and $\mathrm{H}_{2} \mathrm{O}_{2}$-treated human umbilical vein endothelial cells (HUVECs). The preeclamptic Sprague-Dawley (SD) rat model was established by injection of L-NAME and treatment with astaxanthin. The activities of malondialdehyde (MDA), superoxide dismutase (SOD) and nitric oxide synthase (NOS) in serum were analyzed. Pathological changes were examined by hematoxylin and eosin (H\&E) staining. The expression of nuclear factor (NF)- $\mathrm{kB}$, Rho-associated protein kinase II (ROCK II), heme oxygenase-1 (HO-1) and caspase 3 in preeclamptic placentas were examined by immunohistochemistry. Astaxanthin significantly reduced $\mathrm{H}_{2} \mathrm{O}_{2}$-induced HUVEC cell death, decreased ROS and increased MMP. Astaxanthin significantly reduced blood pressure and the content of MDA, but significantly increased the activity of SOD in preeclamptic rats. The urinary protein and the level of $\mathrm{NO}$ and NOS were also decreased. H\&E staining revealed that the thickness of the basilar membrane was increased, while the content of trophoblast cells and spiral arteries were reduced following astaxanthin treatment. Immunohistochemistry results showed that the expression of NF- $\mathrm{KB}$, ROCK II and caspase 3 in preeclamptic placentas was significantly decreased
\end{abstract}

Correspondence to: Dr Hai-Min Chen, School of Marine Science, Ningbo University, 818 Fenghua Road, Ningbo, Zhejiang 315211, P.R. China

E-mail: haiminch75@hotmail.com

Abbreviations: L-NAME, N $\omega$-nitro-L-arginine methyl ester; ROS, reactive oxygen species; MMP, mitochondrial membrane potential; HUVECs, human umbilical vein endothelial cells; SD, sprague-dawley; MDA, malondialdehyde; SOD, superoxide dismutase; NOS, nitric oxide synthase; ROCK II, Rho-associated protein kinase II; HO-1, heme oxygenase-1; PE, preeclampsia; MTT, 3-(4,5-dimethylthiazol-2-yl) 2,5-diphenyltetrazolium bromide

Key words: astaxanthin, preeclampsia, HUVECs, ROS, L-NAME after astaxanthin treatment, while $\mathrm{HO}-1$ expression was increased. In conclusion, astaxanthin inhibited $\mathrm{H}_{2} \mathrm{O}_{2}$-induced oxidative stress in HUVECs. Astaxanthin treatment significantly improved L-NAME-induced preeclamptic symptoms and reduced the oxidative stress and inflammatory damages in preeclamptic placentas. Astaxanthin treatment may effectively prevent and treat preeclampsia.

\section{Introduction}

Preeclampsia (PE) is a common complication during pregnancy, which is characterized by hypertension, high urea protein and abnormalities in other systems (1). PE is a key cause of fatality of pregnant women and perinatal fetuses. It has been demonstrated that PE is associated with immunity, uterus-placental ischemia, endothelin and nitrogen oxide (NO) dysfunction (2); however, the precise etiology of PE remains to be determined. Recent studies demonstrated that PE development is accompanied with reduced placental infusion due to extensive damage in the endothelium, which is predominantly caused by oxidative stress and inflammation $(3,4)$. Moreover, ischemia can induce the production of free radicals and decrease the activity of antioxidative proteins, resulting in damage to the endothelium $(5,6)$. It is well-known that inflammation can cause immune imbalance, endothelial damage, cytokine production, activation of neutrophils and local oxidative stress in the placenta, eventually inducing PE $(6,7)$.

Currently it is difficult to prevent the early stages of PE, clinical treatments predominantly focus on relieving the symptoms, such as reducing blood pressure and seizures, and supplying albumin (8). However, the therapeutic effects of these treatments are not satisfactory and over supplementation of albumin can put a strain on the kidney. Thus, development of novel therapeutic agents to treat PE is required. Harma et al (9) reported that supplementation of antioxidants can reduce the incidence of PE by $2 / 3$ in high-risk pregnant women, which provides direction for PE treatment. Raijmakers et al (10) observed that the combination of vitamins $\mathrm{C}$ and $\mathrm{E}$ is a promising prophylactic strategy for prevention of preeclampsia.

Astaxanthin, 3,3'-dihydroxy- $\beta$-carotene-4,4'-dione, is extensively present in aquatic biology. The major feature of astaxanthin is its antioxidative activity is 100-550 times stronger than that of vitamin E. The biological activities of astaxanthin include clearing cellular reactive oxygen species 
(ROS), reducing oxidative stress, inflammation and blood pressure, and increasing NO utilization (11-14). Thus, according to the biological activities of astaxanthin, it was predicted that astaxanthin may effectively reduce PE. Thus, the present study aimed to investigate the effect of astaxanthin on the antioxidative activity of endothelial cells, and assess the therapeutic effects of astaxanthin on rats with $\mathrm{N} \omega$-nitro-L-arginine methyl ester (L-NAME)-induced preeclampsia.

\section{Materials and methods}

Cell lines and cell culture. Human umbilical vein endothelial cells (HUVECs) were obtained from China Center for Type Culture Collection (Wuhan, China). The cells were maintained in minimal essential medium (Gibco; Thermo Fisher Scientific, Inc., Waltham, MA, USA) supplemented with $10 \%$ fetal bovine serum (Gibco; Thermo Fisher Scientific, Inc.), $100 \mathrm{U} / \mathrm{ml}$ penicillin and streptomycin at $37^{\circ} \mathrm{C}$ in a humidified atmosphere of $5 \% \mathrm{CO}_{2}$, and subcultured upon reaching $80 \%$ confluence.

Cell viability assay. Viability of HUVECs following exposure to astaxanthin isolated from Pluvialis algae (Wako Pure Chemical Industries, Ltd., Wako, Japan) and $\mathrm{H}_{2} \mathrm{O}_{2}$ was assessed using an MTT (3-[4,5-dimethylthiazol-2-yl]2,5-diphenyltetrazolium bromide) cytotoxicity assay. Briefly, 50-60\% confluent cell monolayers $\left(5 \times 10^{5} / \mathrm{ml}\right)$ were exposed to different concentrations of astaxanthin $(0.1,1$ or $10 \mu \mathrm{mol} / \mathrm{l})$ for $48 \mathrm{~h}$. Cells were washed for three times and then cultured in medium containing $500 \mu \mathrm{mol} / 1$ or $2 \mathrm{mmol} / 1 \mathrm{H}_{2} \mathrm{O}_{2}$ for 1 or $24 \mathrm{~h}$. Cells were then incubated with $20 \mu \mathrm{l} \mathrm{MTT}$ for $4 \mathrm{~h}$ at $37^{\circ} \mathrm{C}$. After removing MTT, $200 \mu \mathrm{l}$ dimethyl formamide was added. Absorbance readings were taken at $490 \mathrm{~nm}$ using a microplate reader. Results were expressed as the percentage of control (untreated cells).

Determination of ROS production. Intracellular oxidant stress was monitored by measuring the changes in fluorescence after intracellular probe oxidation. HUVECs were treated with different concentrations of astaxanthin $(0.1,1$ or $10 \mu \mathrm{mol} / \mathrm{l})$ for $48 \mathrm{~h}$ and $2 \mathrm{mmol} / 1 \mathrm{H}_{2} \mathrm{O}_{2}$ for $1 \mathrm{~h}$, then trypsinized and washed twice in phosphate-buffered saline (PBS). The fluorometric probe, 2',7'-dichlorofluorescein diacetate (DCFH-DA) $(20 \mu \mathrm{M})$ was added to the cells and incubated at $37^{\circ} \mathrm{C}$ for $45 \mathrm{~min}$. Cells were washed with PBS and ROS measurement was conducted using a FACSCalibur flow cytometer (BD Biosciences, Franklin Lakes, NJ, USA). In total, 10,000 events were counted in each run using CellQuest software (BD Biosciences) and all the experiments were repeated 3 times.

Determination of the mitochondrial membrane potential (MMP) by JC-1 fluorescence. MMP was measured with the lipophilic cationic probe JC-1. HUVECs were treated with different concentrations of astaxanthin for $48 \mathrm{~h}$ and then $2 \mathrm{mmol} / 1 \mathrm{H}_{2} \mathrm{O}_{2}$ for $1 \mathrm{~h}$. Cells were washed twice in PBS and incubated with $2.5 \mu \mathrm{g} / \mathrm{ml} \mathrm{JC}-1$ in the dark for $20 \mathrm{~min}$ at $37^{\circ} \mathrm{C}$. Cells were washed twice with PBS and resuspended in $400 \mu \mathrm{l}$ PBS and analyzed by flow cytometry. A 488-nm filter was used for excitation of JC-1. Emission filters of 535 and $595 \mathrm{~nm}$ were used to quantify the population of HUVECs with green
(JC-1 monomer) and orange (JC-1 aggregates) fluorescence, respectively. All samples were examined by fluorescence microscopy to confirm JC-1 labeling patterns.

Animals and treatments. A total of 120 mature Sprague-Dawley (SD) rats (weight, 250-260 g; age, 75 days) were purchased from Zhejiang Provincial Experimental Animal Center and housed in the animal center of Ningbo University Medical College (Ningbo, China). All rats were maintained under a 12-h light/dark cycle (08:00 AM lights on) and provided with food and water ad libitum. Housing and experimental environments were temperature- and humidity-controlled ( $21 \pm 2^{\circ} \mathrm{C}$ and $\sim 60 \%$ humidity, respectively). All experimental procedures were performed in accordance with the National Institutes of Health Guide for the Care and Use of Laboratory Animals and approved by the Ethical Committee of Animal Use and Protection of Ningbo University (Ningbo, China).

Male rats and female rats in estrous cycle were mated at a ratio of 1:3. The day of appearance of a vaginal plug was regarded as day 1 of pregnancy. All pregnant rats were randomly assigned to the following 5 groups $(n=10)$ : Blank (no treatment), control (L-NAME treatment only) and three astaxanthin groups that were treated with $5,15,25 \mathrm{mg} / \mathrm{kg}$ body weight (bw)/day astaxanthin, respectively, by gavage from day 5 until the end of experiment. The rats in the control and astaxanthin groups were subcutaneously injected with $125 \mathrm{mg} / \mathrm{kg}$ bw/day L-NAME, while the rats in the blank group were injected with same volume of saline. On day 18 , the blood pressure was detected and urinary protein was measured using a urine analyzer (Hitachi, Ltd., Tokyo, Japan). The increased blood pressure and level of urinary proteins indicated successful generation of preeclamptic models (15). On day 21 , the blood was collected via abdominal aorta. The rats were anesthetized using diethyl ether (Sinopharm Chemical Reagent, Co., Ltd., Beijing, China) and sacrificed by blooding via the femoral artery. The placental tissues were collected, fixed in $4 \%$ paraformaldehyde for $24 \mathrm{~h}$ and embedded in paraffin.

Measurement of serum oxidative parameters. The blood was centrifuged at $1,000 \mathrm{x} \mathrm{g}$ for $10 \mathrm{~min}$ at $4^{\circ} \mathrm{C}$ to collect serum. The activity of serum malondialdehyde (MDA), superoxide dismutase (SOD) and nitric oxide synthase (NOS), and the levels of serum NO and endothelin were measured by kits (Nanjing Jiancheng Bioengineering Institute, Nanjing, China) according to the manufacturer's instructions.

Immunohistochemistry. The placental sections (3-4 $\mu \mathrm{m})$ were deparaffinized and rehydrated in a graded series of ethanol. The sections were boiled in $0.01 \mathrm{M}$ sodium citrate buffer ( $\mathrm{pH}$ 6.0) for $15 \mathrm{~min}$ and treated with $3 \% \mathrm{H}_{2} \mathrm{O}_{2}$ in methanol for 10 min to quench endogenous peroxidase. Then the sections were blocked in horse serum for $30 \mathrm{~min}$ and incubated with various anti-mouse monoclonal antibodies: Anti-nuclear factor (NF) $\kappa \mathrm{B}$ (Santa Cruz Biotechnology Inc., Santa Cruz, CA, USA; 1:100; cat. no. sc-8008), anti-Rho-associated protein kinase II (ROCK II; Santa Cruz Biotechnology Inc.; 1:200; cat. no. sc-398519), anti-Caspase 3 (Santa Cruz Biotechnology Inc.; 1:100; cat. no. sc-271759) and goat anti-mouse polyclonal heme oxygenase-1 (HO-1) antibody (Bioss Inc., Woburn, MA, 
USA; 1:200; cat. no. bs-2075R) overnight at $4^{\circ} \mathrm{C}$. The control sections were omitted for primary antibody. The sections were washed with PBS and incubated with biotinylated goat anti-mouse (cat. no. sc-2005) and donkey anti-goat (cat. no. sc-2020) secondary antibodies (Santa Cruz Biotechnology Inc.; 1:200) for $2 \mathrm{~h}$ at room temperature, followed by the incubation with the streptavidin-biotin-peroxidase complex. The sections were stained with a DAB (3,3'-diaminobenzidine) kit (Vector Laboratories, Inc., Burlingame, CA, USA) and counterstained with hematoxylin, dehydrated with ethanol, cleared with xylene and mounted in synthetic resin. Positive staining, which appeared as a brown color, was visualized under a light microscope. The expression of NF- $\mathrm{B}, \mathrm{HO}-1$, Caspase 3 and ROCK II was quantified using by comparing positive-labeled areas and total areas using Image-Pro Plus 7.0 software (Media Cybernetics, Inc., Rockville, MD, USA).

Statistical analysis. The results are expressed as the mean \pm standard error of mean. Statistical analyses were performed using the SPSS software, version 16.0 (SPSS Inc., Chicago, IL, USA). Differences among groups were analyzed by using one-way analysis of variance and Duncan's test for multiple comparisons. $\mathrm{P}<0.05$ was considered to indicate a statistically significant difference.

\section{Results}

Effect of astaxanthin on cell viability in $\mathrm{H}_{2} \mathrm{O}_{2}$-treated HUVECs. HUVECs were treated with two different concentrations of $\mathrm{H}_{2} \mathrm{O}_{2}$ and cell viability was examined by an MTT assay. As shown in Fig. 1, compared with control cells, cell viability was reduced to $80.47 \%$ after treatment with $500 \mu \mathrm{mol} / 1 \mathrm{H}_{2} \mathrm{O}_{2}$ for $24 \mathrm{~h}$, and to $42.22 \%$ after treatment with $2.0 \mathrm{mmol} / 1 \mathrm{H}_{2} \mathrm{O}_{2}$ for $1 \mathrm{~h}(\mathrm{P}<0.01)$. However, astaxanthin treatment at $1.0 \mu \mathrm{mol} / \mathrm{l}$ and $10 \mu \mathrm{mol} / 1$ significantly improved cell viability in two concentrations of $\mathrm{H}_{2} \mathrm{O}_{2}$-treated HUVECs, in which cell viability in $500 \mu \mathrm{mol} / 1$ but not $2.0 \mathrm{mmol} / 1 \mathrm{H}_{2} \mathrm{O}_{2}$-treated HUVECs was completed rescued by 1.0 and $10 \mu \mathrm{mol} / 1$ astaxanthin (Fig. 1). Thus, $2.0 \mathrm{mmol} / 1 \mathrm{H}_{2} \mathrm{O}_{2}$ was used to examine the effect of astaxanthin on ROS.

Effect of astaxanthin on $\mathrm{ROS}$ and MMP in $\mathrm{H}_{2} \mathrm{O}_{2}$-treated HUVECs. Compared with control, $\mathrm{H}_{2} \mathrm{O}_{2}$ treatment significantly increased the level of ROS in HUVECs $(\mathrm{P}<0.05)$ (Fig. 2). Astaxanthin treatment at 0.1 and $1.0 \mu \mathrm{mol} / 1$ markedly reduced ROS level in $\mathrm{H}_{2} \mathrm{O}_{2}$-treated HUVECs $(\mathrm{P}>0.05)$, and $10 \mu \mathrm{mol} / 1$ astaxanthin significantly reduced the ROS level in $\mathrm{H}_{2} \mathrm{O}_{2}$-treated HUVECs $(\mathrm{P}<0.05)$. These data indicate that high level of astaxanthin can reduce the level of ROS in HUVECs. The effect of astaxanthin on MMP was also examined in $\mathrm{H}_{2} \mathrm{O}_{2}$-treated HUVECs.

Mitochondria are considered the predominant intracellular source of reactive oxygen species. The decrease in MMP is associated with reduced mitochondrial function. When MMP is high, the fluorescence probe JC-1 gathers in the mitochondrial matrix to form aggregates that fluoresce red, while at low MMP JC-1 monomers fluoresce green. In the present study, the green fluorescence was strong following $\mathrm{H}_{2} \mathrm{O}_{2}$ treatment, suggesting low levels of MMP (Fig. 3). However, astaxanthin treatment gradually

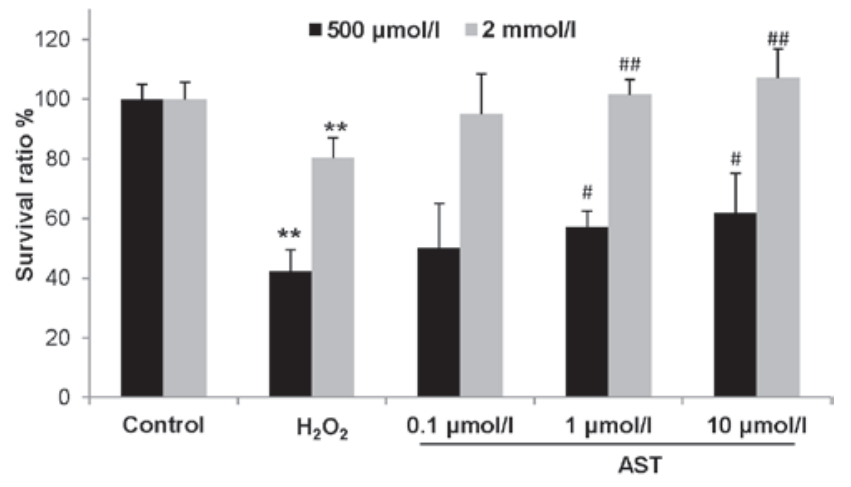

Figure 1. Effect of AST on cell viability of $\mathrm{H}_{2} \mathrm{O}_{2}$-induced human umbilical vein endothelial cells. ${ }^{* *} \mathrm{P}<0.01$ vs. the control group; ${ }^{\#} \mathrm{P}<0.05,{ }^{\# \#} \mathrm{P}<0.01$ vs. the $\mathrm{H}_{2} \mathrm{O}_{2}$ group. $\mathrm{n}=3$. AST, astaxanthin.

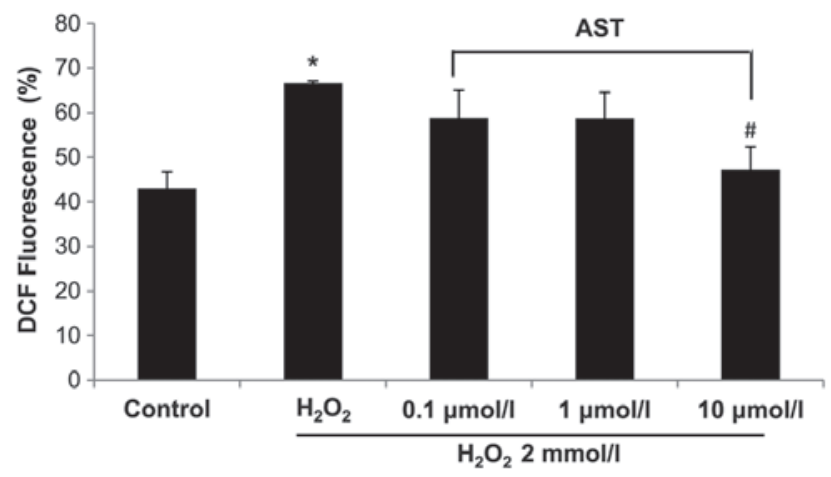

Figure 2. Effect of astaxanthin on reactive oxygen species level in $\mathrm{H}_{2} \mathrm{O}_{2}$-induced human umbilical vein endothelial cells. ${ }^{*} \mathrm{P}<0.05$ vs. the control group; ${ }^{\text {}} \mathrm{P}<0.05$ vs. the $\mathrm{H}_{2} \mathrm{O}_{2}$ group. $\mathrm{n}=3$. AST, astaxanthin.

reduced green fluorescence but increased red fluorescence in $\mathrm{H}_{2} \mathrm{O}_{2}$-treated HUVECs (Fig. 3). These data suggest that astaxanthin treatment can effectively protect MMP in a concentration-dependent manner.

Effect of astaxanthin on blood pressure and urinary protein in preeclamptic rats. L-NAME is the conventional agent used to establish animal models of hypertension. After injection with L-NAME, the systolic and diastolic blood pressures were increased by $25.75(\mathrm{P}<0.05)$ and $23.00 \%$, respectively. Moreover, the urinary protein was increased. After treatment with different concentrations of astaxanthin, blood pressure and urinary protein were reduced in a concentration-dependent manner. Notably, compared with the L-NAME group, $25 \mathrm{mg} / \mathrm{kg}$ bw/day astaxanthin reduced systolic and diastolic blood pressure by 38.09 and $57.80 \%(\mathrm{P}<0.01)$, respectively, which was even significantly lower than that of the blank group $(\mathrm{P}<0.05$, Table I). Urinary protein was also reduced by $25 \mathrm{mg} / \mathrm{kg}$ bw/day astaxanthin.

Effect of astaxanthin on serum parameters in preeclamptic rats. In L-NAME-treated preeclamptic rats, serum $\mathrm{NO}$ and SOD were significantly reduced $(\mathrm{P}<0.05)$, while serum NOS and MDA was markedly changed ( $P>0.05$, Table II). However, $15 \mathrm{mg} / \mathrm{kg}$ bw/day and higher levels of astaxanthin treatment significantly decreased serum MDA but increased 
Table I. Effect of astaxanthin on blood pressure and proteinuria in rats.

\begin{tabular}{lccc}
\hline Group & $\begin{array}{c}\text { Systolic blood } \\
\text { pressure }(\mathrm{mmHg})\end{array}$ & $\begin{array}{c}\text { Diastolic blood } \\
\text { pressure (mmHg) }\end{array}$ & $\begin{array}{c}\text { Urinary protein (pg/ml) } \\
\text { Blank }\end{array}$ \\
L-NAME & $83.50 \pm 9.11$ & $56.50 \pm 10.60$ & $10.51 \pm 1.56$ \\
Astaxanthin (5 mg/kg bw/day) & $105.00 \pm 11.80^{\mathrm{a}}$ & $69.50 \pm 12.34$ & $11.02 \pm 0.79$ \\
Astaxanthin $(15 \mathrm{mg} / \mathrm{kg} \mathrm{bw/day)}$ & $98.30 \pm 5.72$ & $61.05 \pm 3.35$ & $10.84 \pm 1.54$ \\
Astaxanthin $(25 \mathrm{mg} / \mathrm{kg}$ bw/day) & $85.00 \pm 3.88^{\mathrm{b}}$ & $45.26 \pm 2.87^{\mathrm{c}, \mathrm{d}}$ & $10.69 \pm 1.37$ \\
\hline
\end{tabular}

Data are presented as the mean \pm standard deviation $(\mathrm{n}=10)$. ${ }^{\mathrm{a}} \mathrm{P}<0.05$ vs. the blank group; ${ }^{\mathrm{b}} \mathrm{P}<0.05,{ }^{\mathrm{c}} \mathrm{P}<0.01$ vs. the L-NAME group; ${ }^{d} \mathrm{P}<0.01$ vs. the blank group. L-NAME, $\mathrm{N} \omega$-nitro-L-arginine methyl ester; bw, body weight.

Table II. Effect of astaxanthin on the content of NO and MDA, and the activity of NOS and SOD in rat serum.

\begin{tabular}{|c|c|c|c|c|}
\hline Group & $\mathrm{NO}(\mathrm{pg} / \mathrm{ml})$ & NOS $(\mathrm{U} / \mathrm{ml})$ & $\operatorname{MDA}(\mathrm{nmol} / \mathrm{ml})$ & $\mathrm{SOD}(\mathrm{U} / \mathrm{ml})$ \\
\hline Blank & $6.29 \pm 3.57$ & $37.08 \pm 4.27$ & $1.81 \pm 1.59$ & $25.45 \pm 4.64$ \\
\hline L-NAME & $4.74 \pm 2.77^{\mathrm{a}}$ & $35.87 \pm 4.97$ & $1.96 \pm 1.80$ & $16.52 \pm 2.77^{\mathrm{a}}$ \\
\hline Astaxanthin (5 mg/kg bw/day) & $4.76 \pm 2.53$ & $36.05 \pm 3.26$ & $1.84 \pm 0.42$ & $20.58 \pm 6.72$ \\
\hline Astaxanthin (15 mg/kg bw/day) & $4.85 \pm 1.36$ & $36.78 \pm 5.15$ & $1.28 \pm 0.70^{\mathrm{b}}$ & $25.57 \pm 3.83^{\mathrm{b}}$ \\
\hline Astaxanthin (25 mg/kg bw/day) & $4.90 \pm 1.93$ & $37.35 \pm 4.06$ & $0.84 \pm 0.56^{\mathrm{a}, \mathrm{b}}$ & $33.34 \pm 8.16^{\mathrm{a}, \mathrm{b}}$ \\
\hline
\end{tabular}

Data are presented as mean \pm standard deviation $(\mathrm{n}=10)$. ${ }^{\mathrm{a}} \mathrm{P}<0.05$ vs. the blank group, ${ }^{\mathrm{b}} \mathrm{P}<0.05$ vs. the L-NAME group. L-NAME, $\mathrm{N} \omega$-nitro-L-arginine methyl ester; NOS, nitric oxide synthase; MDA, malondialdehyde; SOD, superoxide dismutase; bw, body weight.
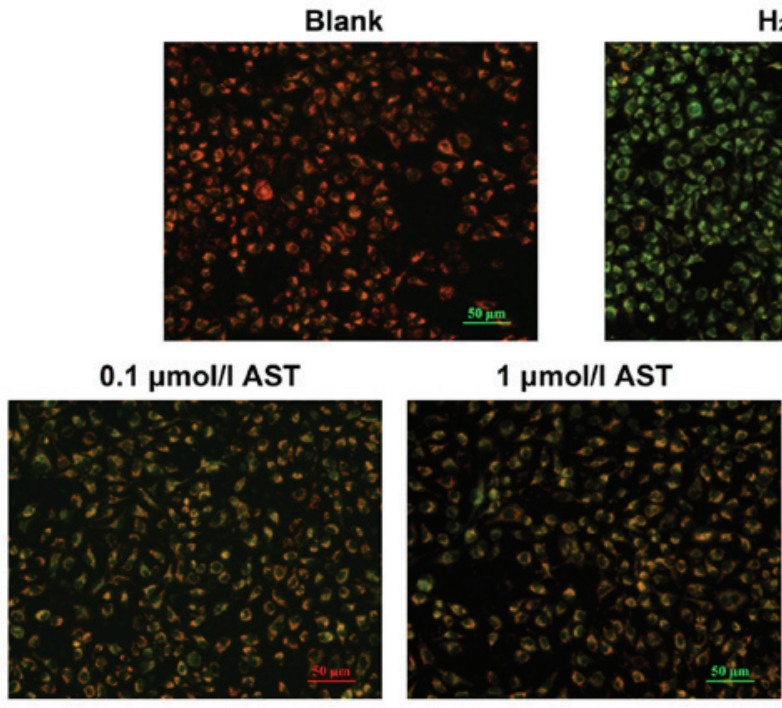

$\mathrm{H}_{2} \mathrm{O}_{2}$

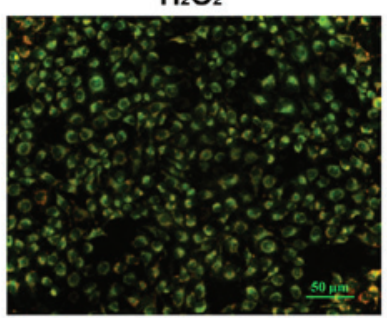

$10 \mu \mathrm{mol} / \mathrm{l} \mathrm{AST}$

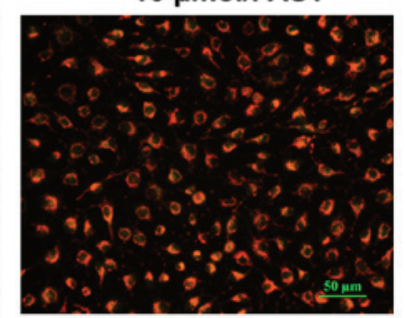

Figure 3. AST regulates mitochondrial membrane potential in $\mathrm{H}_{2} \mathrm{O}_{2}$-treated human umbilical vein endothelial cells. Samples were observed by fluorescence microscopy. The fluorescent images in the green and red channels were captured, and images under the two channels were merged together. AST, astaxanthin.

SOD in preeclamptic rats. Moreover, following treatment with $25 \mathrm{mg} / \mathrm{kg}$ bw/day astaxanthin, serum MDA was decreased while serum SOD level was increased compared with that in the blank group $(\mathrm{P}<0.05)$, while serum NO and NOS were not identified to be significantly changed $(\mathrm{P}>0.05)$.

Effect of astaxanthin on histological changes in preeclamptic rats. The histological changes in placental tissues were also determined following treatment with $25 \mathrm{mg} / \mathrm{kg}$ bw/day astaxanthin in preeclamptic rats. In preeclamptic placental tissues, a few spiral arteries with large lumen and thin vessel walls were observed (Fig. 4). The basement membrane of placental tissue was irregularly thickened with increased cell nodules (Fig. 4). Following treatment with astaxanthin, the number of spiral arteries was decreased compared with the control group, and a few trophoblasts were identified around spiral arteries (Fig. 4). 


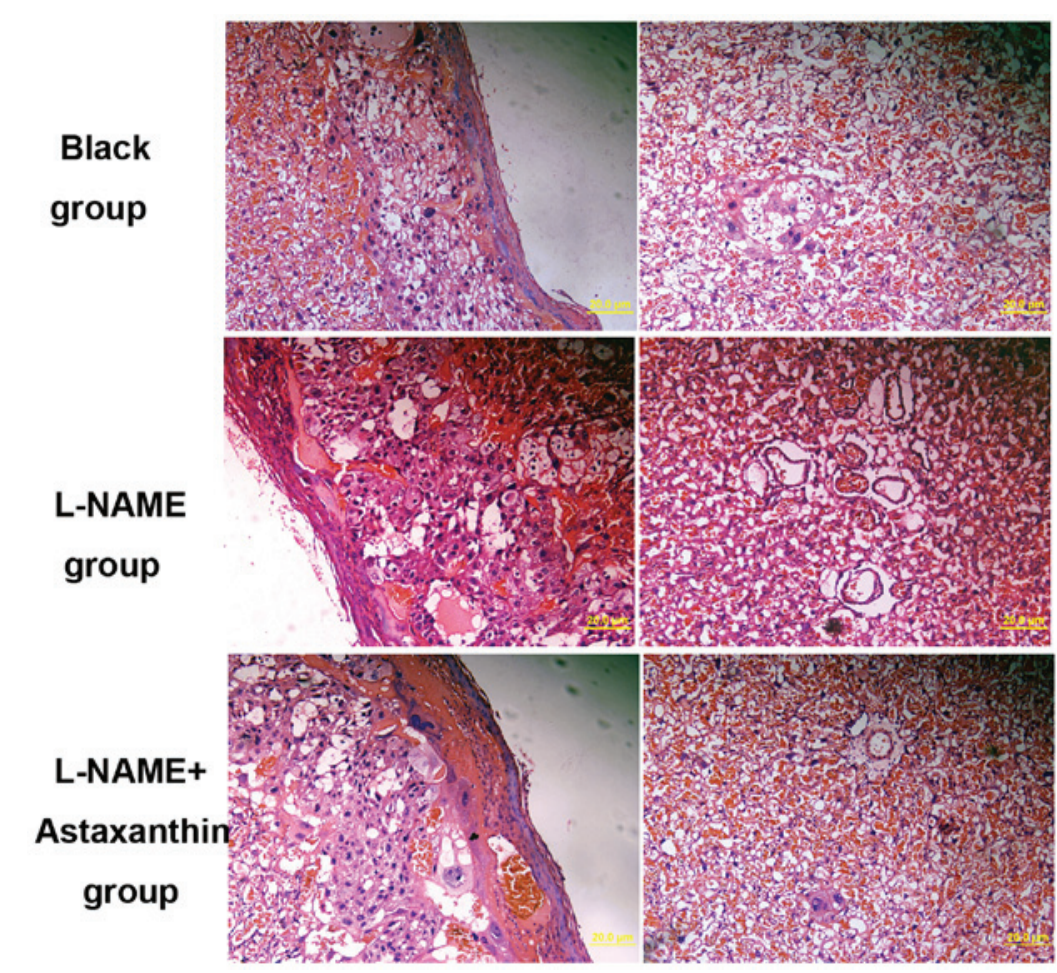

Figure 4. Effect of astaxanthin on the pathological changes in rat placentas. The sections were stained by hematoxylin and eosin staining. Magnification, x100. L-NAME, N $\omega$-nitro-L-arginine methyl ester.
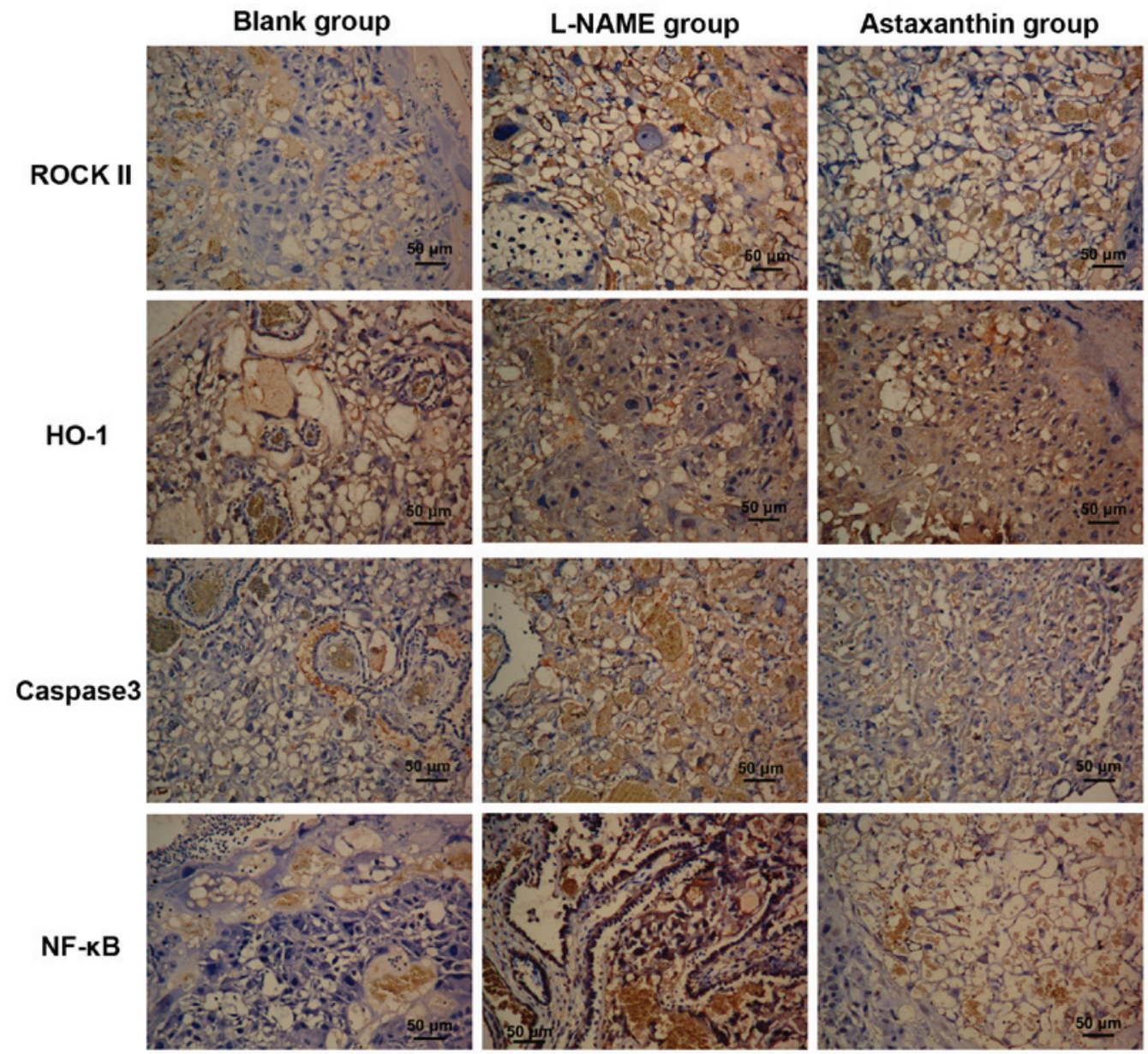

Figure 5. Effect of astaxanthin on the expression ROCK II, HO-1, caspase 3 and NF- $\mathrm{kB}$ in the rat placentas. The sections were stained by immunohistochemistry and DAB staining and nuclei were stained with hematoxylin. The positive signals are in brown color. L-NAME, N $\omega$-nitro-L-arginine methyl ester; ROCK

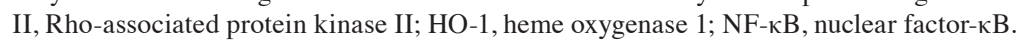


Table III. Effect of astaxanthin on the expression of preeclampsia-associated proteins in the placenta.

\begin{tabular}{lcccc}
\hline Group & ROCK II & HO-1 & Caspase 3 & NF-אB \\
\hline Blank & $260.69 \pm 60.50$ & $6484.38 \pm 638.70$ & $1601.96 \pm 388.09$ & $1748.65 \pm 592.34$ \\
L-NAME & $1002.44 \pm 141.75^{\mathrm{a}}$ & $1893.16 \pm 392.74^{\mathrm{a}}$ & $4253.50 \pm 611.60^{\mathrm{a}}$ & $5110.65 \pm 276.43^{\mathrm{a}}$ \\
Astaxanthin $(25 \mathrm{mg} / \mathrm{kg}$ bw/day) & $678.37 \pm 110.03^{\mathrm{a}, \mathrm{b}}$ & $4875.39 \pm 638.70^{\mathrm{c}}$ & $3025.32 \pm 366.26^{\mathrm{a}}$ & $3607.98 \pm 859.52^{\mathrm{a}, \mathrm{b}}$ \\
\hline
\end{tabular}

Data are presented as the mean \pm standard deviation $(\mathrm{n}=10) .{ }^{\mathrm{a}} \mathrm{P}<0.05$ vs. the blank group; ${ }^{\mathrm{b}} \mathrm{P}<0.05,{ }^{\mathrm{c}} \mathrm{P}<0.01$ vs. the L-NAME group. L-NAME, $\mathrm{N} \omega$-nitro-L-arginine methyl ester; ROCK II, Rho-associated protein kinase II; HO-1, heme oxygenase-1; NF- $\kappa \mathrm{B}$, nuclear factor- $\mathrm{B}$; bw, body weight.

Thus, astaxanthin treatment can significantly improve the pathological changes in preeclamptic rats.

Effect of astaxanthin on the expression of certain proteins in preeclamptic rats. The expression of PE-associated proteins in placental tissues by immunohistochemistry. In the control and astaxanthin groups, ROCK II protein was expressed in the cytoplasm of cytotrophoblasts, syncytiotrophoblasts, endothelial cells and stromal cells, with high expression in the cytoplasm of trophoblasts (Fig. 5). Moreover, after quantitative analysis, ROCK II expression in the control and astaxanthin groups was significantly higher than that in the blank group $(\mathrm{P}<0.01)$, while its expression in the astaxanthin group was significantly lower than that in the control group $(\mathrm{P}<0.05$, Table III).

HO-1 was expressed in the cytoplasm of villous syncytiotrophoblasts and endothelial cells, with low expression in the stromal cells (Fig. 5). Based on the quantitative results, HO-1 expression in the control group was significantly lower than that in the blank group $(\mathrm{P}<0.01)$, astaxanthin treatment significantly increased HO-1 expression in placental tissue compared with the L-NAME group $(\mathrm{P}<0.01)$, however, its expression still lower than that in the blank group. .

Caspase 3 was expressed in the membrane and cytoplasm of trophoblasts (Fig. 5). Its expression was significantly increased in preeclamptic placentas compared with the blank group $(\mathrm{P}<0.01)$, while astaxanthin treatment significantly decreased Caspase 3 expression in the placental tissues $(\mathrm{P}<0.05)$.

$\mathrm{NF}-\kappa \mathrm{B}$ was predominantly expressed in the cytotrophoblasts, with low expression in villous stromal cells. In the control group, high NF- $\mathrm{KB}$ expression was observed in the trophoblasts, with a degree of nucleus staining (Fig. 5). Compared with the blank group, NF- $\mathrm{KB}$ expression was significantly increased in the control group $(\mathrm{P}<0.01)$, while its expression in the astaxanthin group was significantly lower than that in the control group $(\mathrm{P}<0.05)$ but significantly higher than that in the blank group $(\mathrm{P}<0.01)$.

\section{Discussion}

$\mathrm{PE}$ is a specific disorder associated with hypertension during gestation. The pathological mechanisms for PE are complex; however, in recent years, PE has been shown to be associated with the damage of endothelial cells, oxidative stress and inflammation in the placenta (2-6). Abnormal oxidative stress to pregnant women is a key factor involved in the induction of PE.
If placental lesions (such as less placenta blood infusion) affect maternal lipid metabolism to result in uncontrolled lipid peroxidation, they will provide excessive active oxygen and induce oxidative stress, and result in the damage to vessel endothelial cells in the placenta, which will cause pathological damage to placenta and induce PE $(16,17)$. Excessive inflammation has been observed in the early stages of PE and the production of inflammation-related chemokines and cytokines is markedly increased (18). Moreover, a previous study suggested that the development and progression of $\mathrm{PE}$ is the consequence of inflammation and oxidative stress to vessel endothelial cells (19). Thus, antioxidative therapy has been applied in PE treatment (20). Serdar et al (21) found that the level of vitamin E and carotene in patients with severe PE patients is markedly lower than that in pregnant women, suggesting that antioxidant supplements at the early stages of gestation is key in the prevention of PE. Poston et al (22) reported that the incidence of PE in pregnant women taking vitamin $\mathrm{E}$ and $\mathrm{C}$ supplements is lower than that in control and placebo groups. Antioxidative therapy to prevent PE has been performed in the United States, Canada, Mexico, England and other countries, may be particularly efficacious for PE treatment (23).

Astaxanthin is an antioxidant and the strongest known singlet-oxygen quencher (11). Moreover, astaxanthin has an anti-inflammatory effect through inhibiting the expression of inflammation-associated genes and changing the ratio of Th1/Th2 cells $(12,24)$. Additionally, it was reported that astaxanthin can decrease blood pressure in hypertensive mice and increase the utility of NO $(13,25)$. Based on the effects of astaxanthin, it is reasonable to assume that astaxanthin may have therapeutic efficacy in PE, although its application for PE therapy has never been reported.

The polyene structure of astaxanthin leads to low polarity, and it predominantly exists in a crystal form. This results in low permeabilization into cells and thus few studies have been conducted on its effects. However, Chew et al (26) found that astaxanthin does slowly permeate into cells, with a peak at 24-48 h, and thus may be a treatment candidate. In this study, $\mathrm{H}_{2} \mathrm{O}_{2}$ was used to induce oxidative damage and astaxanthin treatment for $48 \mathrm{~h}$ was shown to effectively relieve $\mathrm{H}_{2} \mathrm{O}_{2}$-induced endothelial cell death, reduce the production of active oxygen, and protect the MMP. Astaxanthin treatment significantly increased MMP in $\mathrm{H}_{2} \mathrm{O}_{2}$-treated HUVECs, suggesting that it can effectively decrease the production of ROS, rescue active oxygen-induced oxidative damage to the membrane, and thus protect the function of mitochondria. 
Consistent with a previous study (13), astaxanthin treatment significantly decreased blood pressure and urinary protein in L-NAME-induced preeclamptic rats. The blood pressure in astaxanthin-treated rats was even lower than that in healthy pregnant rats, thus it requires further assessment whether astaxanthin treatment induces low blood pressure. According to the histological data, astaxanthin treatment significantly decreased the number of spiral arteries, villous microvessels and cell nodules in syncytiotrophoblasts, and reversed irregular thickening of the base membrane of trophoblasts. These data indicate astaxanthin treatment can effectively reduce the symptoms of $\mathrm{PE}$ in rats.

Astaxanthin treatment was shown to reduce MDA and increase SOD activity, which supports the major role of astaxanthin treatment in antioxidation. Additionally, NO is a strong vasodilator, which is involved in regulating vascular tone and maintaining hemodynamic equilibrium during gestation. In preeclamptic conditions, the dysfunction of endothelial cells decreases the activity of NOS and the production of NO, and thus causes hypertension (13). In L-NAME-induced preeclamptic rats, serum NOS and NO were markedly decreased; however, astaxanthin treatment only marginally increased serum NOS and NO. Thus, the role of astaxanthin treatment in mediating serum NOS and NO requires further investigation.

It has been demonstrated that $\mathrm{NF}-\kappa \mathrm{B}$ is involved in the regulation of immunity and inflammation (27). In patients with severe $\mathrm{PE}$, increased $\mathrm{NF}-\kappa \mathrm{B}$ expression in placenta tissue affects the invasive capacity of trophoblasts, causes the damage to endothelial cells and induces the production of inflammatory cytokines (28). It was demonstrated that astaxanthin treatment significantly suppressed overexpression of $\mathrm{NF}-\kappa \mathrm{B}$ L-NAME-induced preeclamptic rats. These results indicate that astaxanthin treatment may decrease the production of inflammatory cytokines and the damage to endothelial cells. The Rho/ROCK signaling pathway is important in the regulation of trophoblast proliferation, apoptosis and invasion (29), and ROCK II is regarded as an important effector downstream of the Rho signaling pathway. ROCK II expression is significantly elevated in patients with severe PE, suggesting it may be involved in the development of PE (30). The present study also observed high ROCK II expression in L-NAME-treated rats, but astaxanthin treatment significantly neutralized L-NAME-induced ROCK II expression. These data suggest that astaxanthin may mediate the invasive capacity of trophoblasts through inhibiting ROCK II expression, and inhibiting the progression of PE. HO-1 is a rate-limiting enzyme that catalyzes oxidative degradation of cellular heme to liberate free iron, carbon monoxide (CO) and biliverdin in mammalian cells (31). In addition, its role in heme catabolism, HO-1 exhibits anti-oxidative and anti-inflammatory functions via the actions of biliverdin and $\mathrm{CO}$, respectively (31). Compared with healthy pregnant women, HO-1 expression is significantly reduced in patients with pregnancy-induced hypertension. The present study demonstrated that L-NAME treatment significantly reduced HO-1 expression, while astaxanthin treatment significantly increased HO-1 expression in L-NAME-induced preeclamptic rats. These results suggest that astaxanthin treatment may degrade aging hemoglobin, inhibit antioxidative damage and promote $\mathrm{CO}$ production. It has been demonstrated that oxidative stress and inflammation can cause the apoptosis of trophoblasts and endothelial cells (32). In the present study, it was demonstrated that L-NAME treatment significantly increased caspase 3 expression, while astaxanthin treatment could decrease caspase 3 expression in preeclamptic rats, suggesting that astaxanthin can alleviate apoptosis in PE.

In conclusion, astaxanthin has an antioxidative effect in endothelial cells. Early application of astaxanthin can effectively decrease L-NAME-induced high blood pressure, urinary protein, oxidative stress, inflammation and apoptosis in the placenta. Thus, astaxanthin can reduce the damage to endothelial cells and improve the symptoms of PE; however, its safety in pregnant women requires further investigation.

\section{Acknowledgements}

This study was financed by grants from the Zhejiang Medical Technology Project (grant no.2013KYB238) and the K.C. Wong Magna Fund of Ningbo University, Zhejiang 151 Talents Project.

\section{References}

1. Sibai B, Dekker G and KuPerminc M: Pre-eclampsia. Lancet 365: 785-799, 2005.

2. Redman CW and Sargent IL: Latest advances in understanding preeclampsia. Science 308: 1592-1594, 2005.

3. Vanderlelie J, Gude N and Perkins AV: Antioxidant gene expression in preeclamptic placentae: A preliminary investigation. Placenta 29: 519-522, 2008.

4. Biondi C, Pavan B, Lunghi L, Fiorini S and Vesce F: The role and modulation of the oxidative balance in pregnancy. Curr Pharm Des 11: 2075-2089, 2005

5. Dekker GA and Sibai BM: Etiology and pathogenesis of preeclampsia: Current concepts. Am J Obstet Gynecol 179: 1359-1375, 1998.

6. Var A, Yildirim Y, Onur E, Kuscu NK, Uyanik BS, Goktalay K and Guvenc Y: Endothelial dysfunction in preeclampsia. Increased homocysteine and decreased nitric oxide levels. Gynecol Obstet Invest 56: 221-224, 2003.

7. Germain SJ, Sacks GP, Sooranna SR, Sargent IL and Redman CW: Systemic inflammatory priming in normal pregnancy and preeclampsia: The role of circulating syncytiotrophoblast microparticles. J Immunol 178: 5949-5956, 2007.

8. Magee LA, Miremadi S, Li J, Cheng C, Ensom MH, Carleton B Côté AM and von Dadelszen P: Therapy with both magnesium sulfate and nifedipine does not increase the risk of serious magnesium-related maternal side effects in women with preeclampsia. Am J Obstet Gynecol 193: 153-163, 2005.

9. Harma M, Harma M and Erel O: Oxidative stress in women with preeclampsia. Am J Obstet Gynecol 192: 656-657, 2005.

10. Raijmakers MT, Dechend R and Poston L: Oxidative stress and preeclampsia: Rationale for antioxidant clinical trials. Hypertension 44: 374-380, 2004.

11. Guerin M, Huntley ME and Olaizola M: Haematococcus astaxanthin: Applications for human health and nutrition. Trends Biotechnol 21: 210-216, 2003.

12. Pashkow FJ, Watumull DG and Campbell CL: Astaxanthin: A novel potential treatment for oxidative stress and inflammation in cardiovascular disease. Am J Cardiol 101: 58D-68D, 2008.

13. Hussein G, Goto H, Oda S, Sankawa U, Matsumoto K and Watanabe $\mathrm{H}$ : Antihypertensive potential and mechanism of action of astaxanthin: III. Antioxidant and histopathological effects in spontaneously hypertensive rats. Biol Pharm Bull 29: 684-688, 2006.

14. Ohgami K, Shiratori K, Kotake S, Nishida T, Mizuki N, Yazawa $\mathrm{K}$ and Ohno S: Effects of astaxanthin on lipopolysaccharide-induced inflammation in vitro and in vivo. Invest Ophthalmol Vis Sci 44: 2694-2701, 2003.

15. Takei H, Nakai Y, Hattori N, Yamonoto M, Kurauchi K, Sasaki H and Aburada M: The herbal medicine Toki-shakuyaku-san improves the hypertension and intrauterine growth retardation in preeclampsia rats induced by Nomega-nitro-L-arginine methyl ester. Phytomedicine 11: 43-50, 2004.

16. Bowen RS, Moodley J, Dutton MF and Theron AJ: Oxidative stress in pre-eclampsia. Acta Obstet Gynecol Scan 80: 719-725, 2001. 
17. Tsukimori K, Fukushima K, Tsushima A and Nakano $H$ Generation of reactive oxygen species by neutrophils and endothelial cell injury in normal and preeclamptic pregnancies. Hypertension 46: 696-700, 2005.

18. Redman CW and Sargent IL: Preeclampsia and the systemic inflammatory response. Semin Nephrol 24: 565-570, 2004.

19. Bernardi F, Guolo F, Bortolin T, Petronilho F and Dal-Pizzol F: Oxidative stress and inflammatory markers in normal pregnancy and preeclampsia. J Obstet Gynaecol Res 34: 948-951, 2008.

20. Rumbold A, Duley L, Crowther C and Haslam R: Antioxidants for preventing pr-eclampsia. Cochrane Database Syst Rev CD004227, 2005.

21. Serdar Z, Gür E, Colakoethullarý M, Develioethlu O and Sarandöl E: Lipid and protein oxidation and antioxidant function in women with mild and severe preeclampsia. Arch Gynecol Obstet 268: 19-25, 2003.

22. Poston L, Briley AL, Seed PT, Kelly FJ and Shennan AH; Vitamins in Pre-eclampsia (VIP) Trial Consortium: Vitamin C and vitamin $\mathrm{E}$ in pregnant women at risk for pre-eclampsia (VIP trial): Randomised placebo-controlled trial. Lancet 367: 1145-1154, 2006.

23. Sibai BM: Preeclampsia: An inflammatory syndrome? Am J Obstet Gynecol 191: 1061-1062, 2004.

24. Kidd P: Astaxanthin, cell membrane nutrient with diverse clinical benefits and anti-aging potential. Altern Med Rev 16: 355-364, 2011.
25. Mortensen A, Skibsted LH, Sampson J, Rice-Evans CR and Everett SA: Comparative mechanisms and rates of free radical scaveging by carotenoid antioxidants. FEBS Lett 418: 91-97, 1997.

26. Chew W, Mathison BD, Kimble LL, Mixter PF and Chew BP: Astaxanthin decreases inflammatory biomarkers associated with cardiovascular disease in human umbilical vein endothelial cells. Am J Adv Food Sci Technol 1: 1-175, 2013.

27. Li Q and Verma IM: NF-kappaB regulation in the immune system. Nat Rev Immunol 2: 725-734, 2002.

28. Shah TJ and Walsh SW: Activation of NF-kappaB and expression of COX-2 in association with neutrophil infiltration in systemic vascular tissue of women with preeclampsia. Am J Obstet Gynecol 196: 48. e1-e8, 2007.

29. Pollheimer J and Knöfler M: Signaling pathways regulating the invasive differentiation of human trophoblasts: A review. Placenta 26 (Suppl A): S21-S30, 2005.

30. Ark M, Yilmaz N, Yazici G, Kubat H and Aktas S: Rho-associated protein kinase II (rock II) expression in normal and preeclamptic human placentas. Placenta 26: 81-84, 2005.

31. Appleton SD, Lash GE, Marks GS, Nakatsu K, Brien JF, Smith GN and Graham CH: Effect of glucose and oxygenase depriation on heme oxygenase expression in human chorionic villi explants and immortalized trophoblast cells. Am J Physiol Regul Integr Comp Physiol 285: R1453-R1460, 2003.

32. Myatt L and Cui X: Oxidative stress in the placenta. Histochem Cell Biol 122: 369-382, 2004. 